Check for updates

Cite this: Chem. Commun., 2018, 54,4037

Received 2nd February 2018, Accepted 23rd March 2018

DOI: 10.1039/c8cc00920a

rsc.li/chemcomm

\section{Supramolecular glyco-poly-cyclodextrin functionalized thin-layer manganese dioxide for targeted stimulus-responsive bioimaging $\dagger$}

\author{
Huan Wang, ${ }^{a}$ Ying Liu, ${ }^{a}$ Chao Xu, ${ }^{a}$ Xi Wang, ${ }^{a}$ Guo-Rong Chen, ${ }^{a}$ Tony D. James, (D) \\ Yi Zang, ${ }^{b}$ Jia Li, ${ }^{* b}$ Xiang $\mathrm{Ma}$ (D) ${ }^{* a}$ and Xiao-Peng He (D)*a
}

\begin{abstract}
We have developed a supramoleuclar imaging probe based on thin-layer manganese dioxide functionalized with a fluorescent, multivalent glyco-poly-cycolodextrin for the targeted, stimulusresponsive bioimaging of cancer cells.
\end{abstract}

This paper describes the supramolecular construction of a biocompatible thin-layer manganese dioxide-based probe for the targeted, biothiol-responsive fluorescence imaging of liver and triple-negative breast cancer cells. Target-specific imaging is crucial for improving the precision of diagnostics. Specificity can be achieved through the simultaneous targeting of a membrane receptor and the stimulus-responsive signal production upon interacting with an intracellular biomarker. However, the majority of the current small-molecular probes or polymeric materials for sensing the overexpression of an intracellular disease biomarker lack the ability to be actively internalized by living cells. To address this issue, recent literature has seen an active movement towards the introduction of a targeting agent such as aptamers, peptides and carbohydrates to the imaging material, increasing the accumulation, and thus the stimulus-responsiveness of the probes in a target cell or tissue. ${ }^{1-3}$

There are two key issues that need to be carefully considered to achieve targeted bioimaging. First, multivalent display of the targeting ligands on a material scaffold is crucial for significantly increasing the binding avidity for cell-surface receptors; this is particularly important for carbohydrate-based materials. ${ }^{4}$

\footnotetext{
${ }^{a}$ Key Laboratory for Advanced Materials and Joint International Research Laboratory of Precision Chemistry and Molecular Engineering, Feringa Nobel Prize Scientist Joint Research Center, School of Chemistry and Molecular Engineering, East China University of Science and Technology, 130 Meilong RD, Shanghai 200237, China.E-mail: maxiang@ecust.edu.cn,xphe@ecust.edu.cn

${ }^{b}$ National Center for Drug Screening, State Key Laboratory of Drug Research, Shanghai Institute of Materia Medica, Chinese Academy of Sciences, 189 Guo Shoujing Rd, Shanghai 201203, P. R. China.

E-mail: yzang@simm.ac.cm, jli@simm.ac.cn

${ }^{c}$ Department of Chemistry, University of Bath, Bath, BA2 7AY, UK

$\dagger$ Electronic supplementary information (ESI) available: Additional figures and Experimental section. See DOI: 10.1039/c8cc00920a
}

On a second front, the materials used should be biocompatible in order to achieve real-world applications. Despite the extensive development of oligo- and polymeric architectures to realize multivalency through covalent bonding chemistries, ${ }^{5,6}$ simple yet effective functional imaging probes formed through a noncovalent supramolecular approach remains much less explored.

Here we show the construction of an imaging probe based on 2D manganese dioxide (2D probe) using two supramolecular approaches. Host-guest interaction between an adamantanegrafted fluorescent glycoprobe and poly- $\beta$-cyclodextrins (polyCD) with different CD-grafting ratios (Fig. 1a, where $m$ and $n$ are the equivalent of $\mathrm{CD}$ and that of acrylamide, respectively) forms a multivalent, fluorescence-enhanced glycodot, which can be subsequently self-assembled with a biocompatible 2D material (manganese dioxide - $\mathrm{MnO}_{2}$ ), producing the stimulus-responsive, fluorogenic 2D probe (Fig. 1b) capable of target-specific imaging of liver cancer and triple-negative breast cancer cells (Fig. 1c).

Cyclodextrins (CDs) have been widely used as a macrocyclic host molecule by the supramolecular inclusion of hydrophobic guest compounds in aqueous solution. The supramolecular "host-guest" chemistry of CDs plays an important role in photochemistry, analytical science, materials science and chemical biology. ${ }^{7}$ They have also been used extensively for enhancing drugdelivering efficacy in vivo. ${ }^{8,9}$ With the rapid progress in the discovery of advanced new materials, a myriad of $2 \mathrm{D}$ materials have been synthesized and employed for biosensing and bioimaging. ${ }^{10}$ of the 2D materials developed, thin-layer $\mathrm{MnO}_{2}$ has proven to be rapidly degradable in the presence of reducing agents ${ }^{11}$ such as the physiologically important glutathione (GSH) that exists at higher levels in cancer cells. ${ }^{12,13}$ However, efforts to incorporate the biocompatible poly-CD with $2 \mathrm{D} \mathrm{MnO}_{2}$ producing functional material architectures for biomedical applications remain rare.

To the best of our knowledge, we describe the first supramolecular ensemble formed between functionalized poly-CDs and $2 \mathrm{D} \mathrm{MnO}_{2}$ for targeted, biothiol-responsive fluorescence imaging. We began with the design and synthesis of a naphthalimide glycoprobe (Fig. 1a and Scheme S1, ESI $\dagger$ ). In previous studies, we have shown that the introduction of carbohydrate 


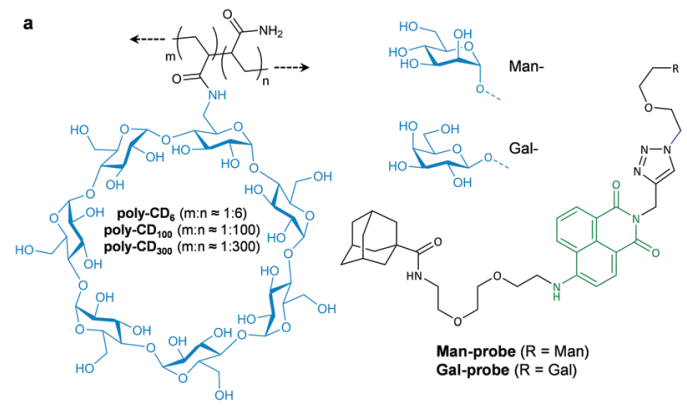

b

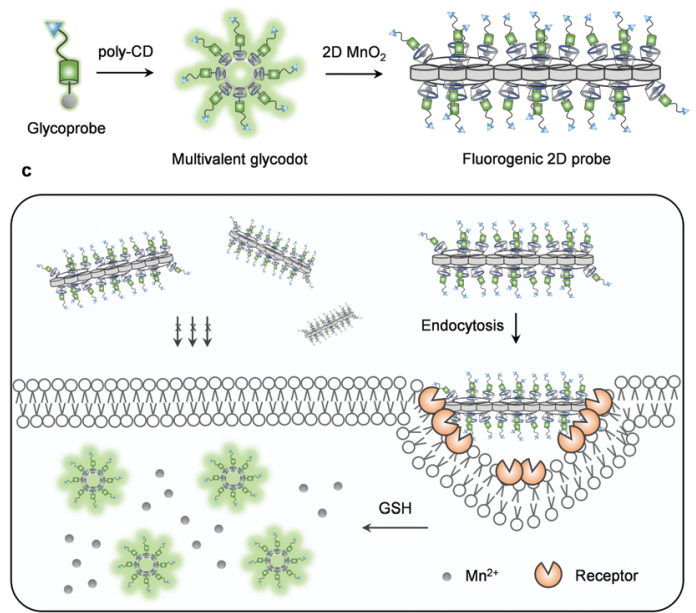

Fig. 1 (a) Structure of the poly-CD host polymers and the adamantanemodified glycoprobes as guest molecules. Schematic illustration of (b) the two-step supramolecular assembly forming the fluorogenic 2D probe and (c) use of the 2D probe for the targeted, stimulus-responsive cell imaging (GSH = glutathione)

targeting agents to naphthalimide based probes significantly enhanced their cell-targeting ability and imaging of intracellular species ${ }^{14}$ with a lowered cytotoxicity. We synthesized two such glyco-naphthalimide probes with galactose and mannose as the targeting ligand for the asialoglycoprotein receptor (ASGPr) and mannose receptor (MR) that exist on the surface of different cancer cells, respectively. An additional adamantane group was introduced to the probes for host-guest assembly with CDs, ${ }^{15,16}$ producing the Man-probe and Gal-probe (Fig. 1a). The CD monomer (6-acrylamido- $\beta$-CD) was synthesized according to the literature ${ }^{17}$ and a subsequent radical binary copolymerization between acrylamide and the CD-monomer with different loading concentrations afforded the poly-CDs with different CD-grafting ratios (poly- $\mathrm{CD}_{6}$, poly- $\mathrm{CD}_{100}$ and poly- $\mathrm{CD}_{300}$, where the numbers refer to the equivalent of acrylamide in the co-polymer).

With the compounds and polymers ready, the self-assembly was carried out in a phosphate buffered saline (PBS, $0.01 \mathrm{M}$, $\mathrm{pH}$ 7.4) as solvent. We determined that the fluorescence of the two glycoprobes enhanced gradually with added poly-CDs (Fig. 2a and d for Man-probe with poly-CD $\mathrm{CD}_{100}$ and Gal-probe with poly- $\mathrm{CD}_{6}$, respectively; for fluorescence spectra of the glycoprobes with all the poly-CDs, see Fig. S1a, ESI $\dagger$ ), suggesting that the host-guest insertion of adamantane into the CD cavities might enhance the hydrophobic environment and thus enhance
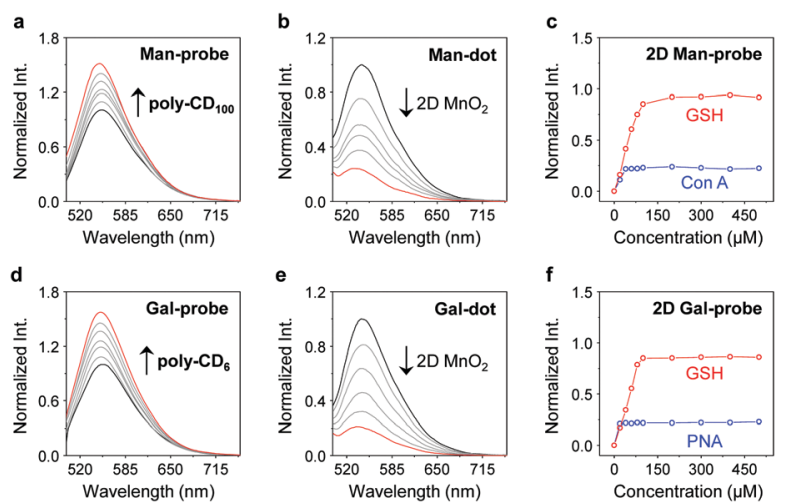

Fig. 2 Concentration-dependent fluorescence enhancement of (a) Manprobe $(1 \mu \mathrm{M})$ with increasing poly- $\mathrm{CD}_{100}\left(0-90 \mu \mathrm{g} \mathrm{mL}^{-1}\right.$; interval: $\left.15 \mu \mathrm{g} \mathrm{mL}^{-1}\right)$ and (d) Gal-probe $(1 \mu \mathrm{M})$ with increasing poly- $\mathrm{CD}_{6}\left(0-90 \mu \mathrm{gL}^{-1}\right.$; interval: $15 \mu \mathrm{g} \mathrm{mL}{ }^{-1}$ ). Concentration-dependent fluorescence quenching of (b) Man-dot (Man-probe/poly- $\mathrm{CD}_{100}=1 \mu \mathrm{M} / 80 \mu \mathrm{g} \mathrm{mL} \mathrm{m}^{-1}$ ) and (e) Galdot (Gal-probe/poly-CD $6=1 \mu \mathrm{M} / 80 \mu \mathrm{gL}^{-1}$ ) with increasing $2 \mathrm{D}^{\mathrm{MnO}_{2}}$ (0-5 $\mu \mathrm{g} \mathrm{mL}{ }^{-1}$; interval: $1 \mu \mathrm{g} \mathrm{mL}{ }^{-1}$ ). Concentration-dependent fluorescence recovery of (c) $2 \mathrm{D}$ Man-probe (Man-probe/poly- $\mathrm{CD}_{100} / 2 \mathrm{D} \mathrm{MnO}_{2}=$ $1 \mu \mathrm{M} / 80 \mu \mathrm{g} \mathrm{mL}^{-1} / 5 \mu \mathrm{g} \mathrm{mL}^{-1}$ ) with increasing GSH and concanavalin $\mathrm{A}$ (Con A, a mannose selective lectin) and (f) 2D Gal-probe (Gal-probe/ poly- $\mathrm{CD}_{6} / 2 \mathrm{D} \mathrm{MnO}_{2}=1 \mu \mathrm{M} / 80 \mu \mathrm{g} \mathrm{mL}^{-1} / 5 \mu \mathrm{g} \mathrm{m}^{-1}$ ) with increasing GSH and peanut agglutinin (PNA, a galactose selective lectin). The fluorescence spectra for glycoprobes and glycodots were obtained in phosphate buffered saline (PBS, $0.01 \mathrm{M}, \mathrm{pH}$ 7.4). The fluorescence spectra for $2 \mathrm{D}$ probes were obtained in Tris- $\mathrm{HCl}(0.01 \mathrm{M}, \mathrm{pH}$ 7.4). Excitation wavelength: $450 \mathrm{~nm}$; slit widths ex $=5 \mathrm{~nm}$ and $\mathrm{em}=5 \mathrm{~nm}$.

the fluorescence emission. ${ }^{18,19}$ We then observed that the enhanced fluorescence intensity of the probes was proportional to the $\mathrm{CD}$ density of the polymers (poly- $\mathrm{CD}_{6}>$ poly- $\mathrm{CD}_{100}>$ poly- $\mathrm{CD}_{300}$ ) (Fig. S1b, ESI $\dagger$ ). This suggests that the polymers with a higher CD density can host more guest molecules to enhance the overall fluorescence emission. To corroborate that the fluorescence enhancement was the result of host-guest interactions, we used 1-bromonaphthalene as a competing molecule. The results indicated that the presence of 1-bromonaphthalene decreased the fluorescence of the glycoprobe/poly-CD ensembles (glycodots), suggesting the interruption of the adamantane-CD binding (Fig. S2, ESI $\dagger$ ).

Having developed the glycodots, we set out to further construct the $2 \mathrm{D}$ probe. The glycodots were mixed with an aqueous dispersion of $2 \mathrm{D} \mathrm{MnO}_{2}$ (with a size distribution in the nanometer range - Fig. S3, ESI $\dagger$ ) produced using an established method. ${ }^{20}$ Then, the assembly was measured by fluorescence spectroscopy. We observed a concentration-dependent fluorescence decrease of the glycodots with $2 \mathrm{D} \mathrm{MnO}_{2}$ (Fig. 2b and e for a Man-dot and a Gal-dot, respectively), which is in agreement with the fluorescence quenching property of the 2D material for surface-bound fluorophores. ${ }^{21,22}$ The quenching plateau was reached with $5 \mu \mathrm{g} \mathrm{mL}{ }^{-1}$ of the $2 \mathrm{D}$ material. The $2 \mathrm{D}$ probes were shown to be thermally stable over a temperature range of $0-200{ }^{\circ} \mathrm{C}$ as determined by thermogravimetric analysis. (Fig. S4, ESI $\dagger$ ).

Next, we tested the stability of the $2 \mathrm{D}$ probes with both lectins (proteins that selectively recognize carbohydrates) and GSH. Interestingly, whereas the presence of selective lectins 
(concanavalin A and peanut agglutinin for mannose- and galactose-based glycodots, respectively) caused minimal fluorescence recovery, the addition of GSH completely restored the fluorescence of the glycodots (Fig. 2c and f for 2D Man-probe and 2D Gal-probe, respectively; for original fluorescence spectra, see Fig. S5, ESI $\dagger$ ). This implies that the poly-CD probes are immobilized on the surface of $2 \mathrm{D} \mathrm{MnO}_{2}$, whereas the degradation of the substrate material by $\mathrm{GSH}^{23}$ releases the fluorescent polymers, thus producing a stimulus-responsive, fluorogenic $2 \mathrm{D}$ probe. The stability of the $2 \mathrm{D}$ probe is important since we envision that the glycodots on the material surface would remain integrated upon receptor endocytosis at the cellular level but become activated only when a target intracellular biomarker for material disruption exists, thereby improving the imaging accuracy.

With these promising solution-based results in hand, we evaluated the imaging ability of the $2 \mathrm{D}$ probes. We first used the glycodots consisting of different polymer backbones and glycoprobes to optimize the imaging condition. Two cancer cell lines, i.e. a triple-negative breast cancer cell line (MDA-MB-231) that overly expresses $\mathrm{MR}^{24}$ and a liver cancer cell line (Hep-G2) that overly expresses $\mathrm{ASGPr}^{25}$ were used. We determined that the density in CD fraction of the poly-CDs had a significant impact on the imaging efficiency. The poly- $\mathrm{CD}_{100}$ and poly- $\mathrm{CD}_{6}$ scaffold produced the highest imaging brightness for MDA-MB-231 and Hep-G2 cells, respectively, among other control poly-CDs (Fig. S6, $\mathrm{ESI} \dagger$ ). This suggests the sharp difference in preferred multivalent glycostructures of ASGPr and MR probably because of the different membrane-bound architectures of the receptors. ${ }^{26-28}$

To corroborate the receptor-targeting ability of the probes, a competition assay was carried out. The result indicated that the presence of increasing free D-mannose and D-galactose gradually suppressed the fluorescence of poly- $\mathrm{CD}_{100}$ and poly- $\mathrm{CD}_{6}$ probe produced in MDA-MB-231 and Hep-G2 cells, respectively (Fig. S7, ESI $\dagger$ ). In addition, the use of a control cell line (human lung cancer - A549) with minimal ASGPr and MR expression as determined by real-time quantitative polymerase chain reaction (Fig. S8, ESI $\dagger$ ) also confirmed the excellent targeting ability of the multivalent glycodots for the MR-expressing and ASGPrexpressing cells (Fig. 3). We also noted that the selectivity of the glyco-dots for the target cancer cell was much higher than that of the glycoprobes alone (Fig. 3), which could be ascribed to the multivalency of the glycodots to enhance the binding avidity with the selective receptors on the cell surface.

Encouraged by these results, we used the thin-layer $\mathrm{MnO}_{2}$ based 2D probes for GSH-responsive imaging of the target cancer cells. Owing to the presence of a high level of endogenous GSH in cancer cells, ${ }^{29-31}$ the cells were pre-treated with NEM (a GSH quencher) ${ }^{32}$ followed by incubation of exogenous GSH to examine the sensitivity of the probes. We observed that the fluorescence of the 2D probes produced in untreated MDA-MB-231 was almost completely supressed in the cells pre-treated with NEM ( $N$-ethylmaleimide) (Fig. 4). However, the subsequent addition of exogenous GSH further enhanced the fluorescence. These results indicate that (1) the $2 \mathrm{D}$ probe is GSH-responsive due to the intracellular degradation of $2 \mathrm{D} \mathrm{MnO}_{2}$ by GSH found in the cells, and that (2) the $2 \mathrm{D}$ probe does not produce the imaging signal
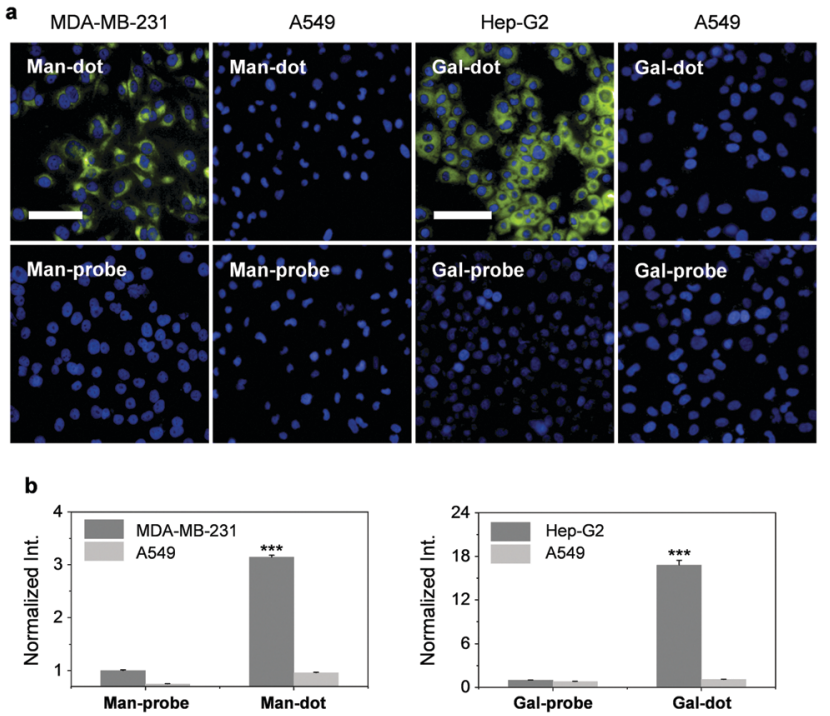

Fig. 3 Fluorescence (a) imaging and (b) quantification ( $* * * P<0.001$ with respect to the group of glycoprobe alone for target cells) of MDA-MB-231 (human triple-negative breast cancer), Hep-G2 (human liver cancer) and A549 (human lung cancer) cells with glycoprobe alone $(8 \mu \mathrm{M})$ or glycodots (glycoprobe/poly-CD $=8 \mu \mathrm{M} / 640 \mu \mathrm{g} \mathrm{mL}{ }^{-1}$ ). Scale bars $=100 \mu \mathrm{m}$. The excitation and emission channels used are 460-490 nm and 530-590 nm, respectively. The cell nuclei were stained by Hoechst 33342.

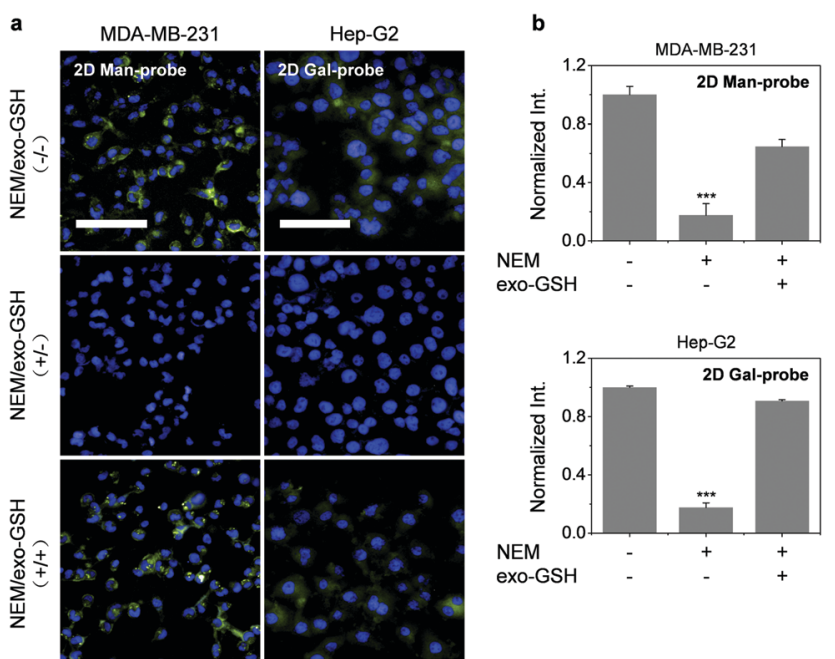

Fig. 4 Fluorescence (a) imaging and (b) quantification ( ${ }^{\star * *} P<0.001$ with respect to the group of untreated cells) of MDA-MB-231 (human triple-negative breast cancer) and Hep-G2 (human liver cancer) cells with or without treatment of NEM or exo-GSH upon incubation with 2D probes (glycoprobe/poly-CD/2D $\mathrm{MnO}_{2}=4 \mu \mathrm{M} / 320 \mu \mathrm{g} \mathrm{mL}-1 / 12 \mu \mathrm{g} \mathrm{mL}{ }^{-1}$ ). Scale bars $=100 \mu \mathrm{m}$. The excitation and emission channels used are 460-490 nm and 530-590 nm, respectively. The cell nuclei were stained by Hoechst 33342.

without intracellular GSH, suggesting good stability of the supramolecular architecture. The second point is particularly important for imaging probes since false-positive signals produced by the non-specific interaction within complex cellular environments could be minimized.

To conclude, we have developed a 2D probe for the targeted, fluorogenic imaging of cancer cells through both host-guest 
interactions and self-assembly between fluorescent polymers and $2 \mathrm{D}$ materials. The simplicity in material construction yet effectiveness of the probes for stimulus-responsive fluorogenic imaging makes it possible to extend these systems for an even wider diversity of supramolecular, biocompatible imaging probes for functional bioimaging.

This research is supported by the National Natural Science Foundation of China (No. 21722801, 21722603 and 21776078), the Programme of Introducing Talents of Discipline to Universities (B16017), the Science and Technology Commission of Shanghai Municipality (15540723800), the Fundamental Research Funds for the Central Universities (222201717003) and the Shanghai Rising-Star Program (16QA1401400 to X.-P. H.). The Catalysis And Sensing for our Environment (CASE) network is thanked for research exchange opportunities. T. D. J. wishes to thank the Royal Society for a Wolfson Research Merit Award and ECUST for a guest professorship.

\section{Conflicts of interest}

The authors have no conflicts of interest to declare.

\section{Notes and references}

1 (a) M. H. Lee, E. J. Kim, H. Lee, H. M. Kim, M. J. Chang, S. Y. Park, K. S. Hong, J. S. Kim and J. L. Sessler, J. Am. Chem. Soc., 2016, 138, 16380-16387; (b) X. Li, C. Y. Kim, S. Lee, D. Lee, H. M. Chung, G. Kim, S. H. Heo, C. Kim, K. S. Hong and J. Yoon, J. Am. Chem. Soc., 2017, 139, 10880-10886; (c) G. C. Van de Bittner, C. R. Bertozzi and C. J. Chang, J. Am. Chem. Soc., 2013, 135, 1783-1795.

2 (a) X.-P. He, Y. Zang, T. D. James, J. Li, G.-R. Chen and J. Xie, Chem. Commun., 2017, 53, 82-90; (b) X.-P. He, Y.-L. Zeng, Y. Zang, J. Li, R. A. Field and G.-R. Chen, Carbohydr. Res., 2016, 429, 1-22.

3 (a) X.-P. He, Y. Zang, T. D. James, J. Li and G.-R. Chen, Chem. Soc. Rev., 2015, 44, 4239-4248; (b) X.-P. He and H. Tian, Small, 2016, 12, 144-160; (c) X.-P. He, X.-L. Hu, T. D. James, J. Yoon and H. Tian, Chem. Soc. Rev., 2017, 46, 6687-6696; (d) X.-P. He and H. Tian, Chem, 2018, 4, 246-268.

4 Z. Li, L. Sun, Y. Zhang, A. P. Dove, R. K. O'Reilly and G. Chen, ACS Macro Lett., 2016, 5, 1059-1064.

5 Z. Chen, Y. Liu, W. Wagner, V. Stepanenko, X. Ren, S. Ogi and F. Wurthner, Angew. Chem., Int. Ed., 2017, 56, 5729-5733.

6 G. Yu, M. Zhang, M. L. Saha, Z. Mao, J. Chen, Y. Yao, Z. Zhou, Y. Liu, C. Gao, F. Huang, X. Chen and P. J. Stang, J. Am. Chem. Soc., 2017, 139, 15940-15949.

7 H. Chen, X. Ma, S. Wu and H. Tian, Angew. Chem., Int. Ed., 2014, 53, 14149-14152.
8 (a) M. J. Webber and R. Langer, Chem. Soc. Rev., 2017, 46, 6600-6620; (b) D. Prochowicz, A. Kornowicz and J. Lewinski, Chem. Rev., 2017, 117, 13461-13501.

9 J. Zhou, G. Yu and F. Huang, Chem. Soc. Rev., 2017, 46, 7021-7053. 10 H. Lin, Y. Wang, S. Gao, Y. Chen and J. Shi, Adv. Mater., 2018, 30.

11 H. Fan, Z. Zhao, G. Yan, X. Zhang, C. Yang, H. Meng, Z. Chen, H. Liu and W. Tan, Angew. Chem., Int. Ed., 2015, 54, 4801-4805.

12 Z. Liu, X. Zhou, Y. Miao, Y. Hu, N. Kwon, X. Wu and J. Yoon, Angew. Chem., Int. Ed., 2017, 56, 5812-5816.

13 Y. Yue, F. Huo, P. Ning, Y. Zhang, J. Chao, X. Meng and C. Yin, J. Am. Chem. Soc., 2017, 139, 3181-3185.

14 (a) K.-B. Li, Y. Zang, H. Wang, J. Li, G.-R. Chen, T. D. James, X.-P. He and H. Tian, Chem. Commun., 2014, 50, 11735-11737; (b) D. T. Shi, D. Zhou, Y. Zang, J. Li, G. R. Chen, T. D. James, X. P. He and H. Tian, Chem. Commun., 2015, 51, 3653-3655; (c) L. Dong, Y. Zang, D. Zhou, X.-P. He, G.-R. Chen, T. D. James and J. Li, Chem. Commun., 2015, 51, 11852-11855; (d) X.-L. Hu, Y. Zang, J. Li, G.-R. Chen, T. D. James, X.-P. He and H. Tian, Chem. Sci., 2016, 7, 4004-4008; (e) K.-B. Li, N. Li, Y. Zang, G.-R. Chen, J. Li, T. D. James, X.-P. He and H. Tian, Chem. Sci., 2016, 7, 6325-6329; $(f)$ X.-P. He, Y.-L. Zeng, X.-Y. Tang, N. Li, D.-M. Zhou, G.-R. Chen and H. Tian, Angew. Chem., Int. Ed., 2016, 55, 13995-13999.

15 L. Wu, Y. Zhang, Z. Li, G. Yang, Z. Kochovski, G. Chen and M. Jiang, J. Am. Chem. Soc., 2017, 139, 14684-14692.

16 Z. Ye, Q. Zhang, S. Wang, P. Bharate, S. Varela-Aramburu, M. Lu, P. H. Seeberger and J. Yin, Chem. - Eur. J., 2016, 22, 15216-15221.

17 A. Harada, R. Kobayashi, Y. Takashima, A. Hashidzume and H. Yamaguchi, Nat. Chem., 2011, 3, 34-37.

18 S. K. Samanta, J. Quigley, B. Vinciguerra, V. Briken and L. Isaacs, J. Am. Chem. Soc., 2017, 139, 9066-9074.

19 P. Wang, C. Zhang, H.-W. Liu, M. Xiong, S.-Y. Yin, Y. Yang, X.-X. Hu, X. Yin, X.-B. Zhang and W. Tan, Chem. Sci., 2017, 8, 8214-8220.

20 K. Kai, Y. Yoshida, H. Kageyama, G. Saito, T. Ishigaki, Y. Furukawa and J. Kawamata, J. Am. Chem. Soc., 2008, 130, 15938-15943.

21 D. K. Ji, Y. Zhang, Y. Zang, J. Li, G. R. Chen, X. P. He and H. Tian, Adv. Mater., 2016, 28, 9356-9363.

22 D.-K. Ji, G.-R. Chen, X.-P. He and H. Tian, Adv. Funct. Mater., 2015, 25, 3483-3487.

23 G. Yang, L. Xu, Y. Chao, J. Xu, X. Sun, Y. Wu, R. Peng and Z. Liu, Nat. Commun., 2017, 8, 902.

24 M. Gary-Bobo, Y. Mir, C. Rouxel, D. Brevet, I. Basile, M. Maynadier, O. Vaillant, O. Mongin, M. Blanchard-Desce, A. Morere, M. Garcia, J. O. Durand and L. Raehm, Angew. Chem., Int. Ed., 2011, 50, 11425-11429.

25 T. Lawrence and G. Natoli, Nat. Rev. Immunol., 2011, 11, 750-761.

26 M. Monestier, P. Charbonnier, C. Gateau, M. Cuillel, F. Robert, C. Lebrun, E. Mintz, O. Renaudet and P. Delangle, ChemBioChem, 2016, 17, 590-594.

27 P. D. Stahl and R. A. B. Ezekowitz, Curr. Opin. Immunol., 1998, 10, 50-55.

28 R. A. Ezekowitz, J. Exp. Med., 1990, 172, 1785-1794.

29 C. X. Yin, K. M. Xiong, F. J. Huo, J. C. Salamanca and R. M. Strongin, Angew. Chem., Int. Ed., 2017, 56, 13188-13198.

30 M. H. Lee, Z. Yang, C. W. Lim, Y. H. Lee, S. Dongbang, C. Kang and J. S. Kim, Chem. Rev., 2013, 113, 5071-5109.

31 J. Yin, Y. Kwon, D. Kim, D. Lee, G. Kim, Y. Hu, J. H. Ryu and J. Yoon, Nat. Protoc., 2015, 10, 1742-1754.

32 K. Umezawa, M. Yoshida, M. Kamiya, T. Yamasoba and Y. Urano, Nat. Chem., 2017, 9, 279-286. 